\title{
STABILITY OF A SCALAR DIFFERENTIAL EQUATION
}

\author{
R. GRIMMER
}

\begin{abstract}
Sufficient conditions are given for the stability and asymptotic stability of the zero solution of a scalar differential equation frequently encountered in the comparison method.
\end{abstract}

1. Consider the scalar differential equation

$$
r^{\prime}=w(t, r) \quad\left({ }^{\prime}=d / d t\right)
$$

where $w(t, r)$ is defined and continuous for $t \geqq 0$ and $0 \leqq r \leqq r_{0}$, for some positive constant $r_{0}$. Under the assumption that $w(t, 0)=0$ for all $t$, sufficient conditions are given for the stability, uniform stability, equiasymptotic stability and uniform asymptotic stability of the zero solution of (1). (For definitions see [3].)

These properties of the scalar equation have been examined previously in the case when (1) can be written in the form

$$
r^{\prime}=\lambda(t) \phi(r)
$$

with $\phi(r)>0$ for $r>0, \phi(0)=0$, by Brauer [1] and in [3] and are of importance in differential inequalities involving Liapunov functions. For an excellent exposition of this method, see [3].

Noting that uniqueness to the right of the zero solution is necessary for stability, we give sufficient conditions for the nonuniqueness of the zero solution of (1). From this result it will be seen that in certain cases the conditions given in [1] and [3] for the stability of the zero solution of (2) are incorrect and so direct proofs will be given for the theorems dealing with the stability of the zero solution of (1).

2. We assume that $w(t, r)$ can be written in the form

$$
w(t, r)=w_{1}(t, r)+w_{2}(t, r)
$$

where $w_{i}(t, r)$ is defined and continuous for $t \geqq 0,0 \leqq r \leqq r_{0}, i=1,2$. Also assume the existence of a continuous function $L(r)$ defined for $0<r \leqq r_{0}$ with $L(r)>0$ and consider the following set of assumptions:

(I) $w_{1}(t, r) L(r)$ is nondecreasing in $r$ for fixed $t$.

(II) $w_{2}(t, r) L(r)$ is nonincreasing in $r$ for fixed $t$.

Received by the editors July 26, 1971.

AMS 1970 subject classifications. Primary 34D20, 34A40, 34A10.

Key words and phrases. Stability, asymptotic stability, nonuniqueness.

(C) American Mathematical Society 1972 
We note that if $w_{i}(t, r) \geqq 0, i=1,2$, that this decomposition of $w(t, r)$ has been used previously by Hallam and Heidel [2], where they considered the boundedness and continuability of the solutions of (1). Also, we note that (2) can be decomposed as above with $L(r)=(1 / \phi(r)), w_{1}(t, r)=0$ and $w_{2}(t, r)=\lambda(t) \phi(r)$.

In addition, we assume

(III) there exists a continuous function $q(t)$ defined for $t \geqq 0$ with $w_{2}(t, r) L(r) \leqq q(t)$ for $t \geqq 0,0<r \leqq r_{0}$.

Further, we shall usually assume

(IV) $\int_{0}^{r_{0}} L(r) d r=\infty$.

We remark that (I), (III), and (IV) imply that the zero solution of (1) is unique. We see this as follows. From (1) and (III),

which we write

$$
w(t, r) \leqq\left(w_{1}\left(t, r_{0}\right) L\left(r_{0}\right)+q(t)\right)(1 / L(r))
$$

$$
w(t, r) \leqq \lambda_{1}(t) \phi_{1}(r),
$$

and from (IV), we see that Osgood's uniqueness criterion is fulfilled for the uniqueness of the zero solution.

THEOREM 1. Let (II) hold and assume that $w_{1}(t, r) \geqq 0$ and $w_{2}(t, r)>0$ for $0 \leqq t_{0}<t \leqq t_{1}, 0<r \leqq r_{0}$. Then (IV) is a necessary condition for the zero solution of (1) to be unique to the right at $t=t_{0}$.

Proof. Suppose (IV) is not valid. We will construct a solution, $r(t)$, of (1) with $r\left(t_{0}\right)=0$ and $r(t)>0$ for $t$ immediately to the right of $t_{0}$.

As $w(t, r)$ is continuous, there exist $\varepsilon>0$ and $t_{2}, t_{1} \geqq t_{2}>t_{0}$, so that if $0 \leqq r_{1} \leqq \varepsilon$ then $r\left(t ; r_{1}, t_{0}\right)$ exists on $\left[t_{0}, t_{2}\right]$ where $r\left(t ; r_{1}, t_{0}\right)$ is any solution of (1) with $r\left(t_{0} ; r_{1}, t_{0}\right)=r_{1}$. Further, there exists $r_{2}, 0<r_{2} \leqq r_{0}$, such that $r\left(t ; r_{1}, t_{0}\right) \leqq r_{2}$ for $t_{0} \leqq t \leqq t_{2}, 0 \leqq r_{1} \leqq \varepsilon$. For each positive integer $n$, choose a solution $r_{n}(t)=r\left(t ; \varepsilon / n, t_{0}\right)$ on $\left[t_{0}, t_{2}\right]$. Choose $r_{3}>0$ with the property that

$$
\int_{0}^{r_{3}} L(r) d r \leqq \int_{t_{0}}^{t_{2}} w_{2}\left(s, r_{2}\right) L\left(r_{2}\right) d s .
$$

For each $n$ we have

$$
\begin{aligned}
\int_{0}^{r\left(t_{2}\right)} L(r) d r & \geqq \int_{\varepsilon / n}^{r\left(t_{2}\right)} L(r) d r \geqq \int_{t_{0}}^{t_{2}} w_{2}(s, r) L(r) d s \\
& \geqq \int_{t_{0}}^{t_{2}} w_{2}\left(s, r_{2}\right) L\left(r_{2}\right) d s \geqq \int_{0}^{r_{3}} L(r) d r .
\end{aligned}
$$

Hence, $r_{n}\left(t_{2}\right) \geqq r_{3}>0$ for every $n$. Now $\left\{r_{n}(t)\right\}$ is a uniformly bounded equicontinuous sequence of functions and by Ascoli's Theorem there is a subsequence which converges uniformly to a solution $r(t)$ of $(1)$ on $\left[t_{1}, t_{2}\right]$. 
As $r_{n}\left(t_{0}\right)=\varepsilon / n$, we see that $r\left(t_{0}\right)=0$ and as $r_{n}\left(t_{2}\right) \geqq r_{3}>0$, we see that $r\left(t_{2}\right)>0$. For $t_{3}$ with $t_{0}<t_{3}<t_{2}$, we see that the above argument shows that we must have $r\left(t_{3}\right)>0$. Hence the zero solution of (1) is not unique to the right and the proof is complete.

REMARK. From Theorem 1 we see that if, in (2), $\lambda\left(t_{0}\right)>0$ and $\int_{0}^{r_{0}}(1 / \phi(r)) d r<\infty$ then the zero solution of (2) is not unique to the right and the zero solution cannot be stable at $t_{0}$, and we see that the results for the stability of the zero solution of (2) given in [1] and [3, p. 139] are incorrect as $\int_{0}^{r_{0}}(1 / \phi(r)) d r<\infty$ was allowed with $\lambda(t)>0$.

As a further application of Theorem 1 , consider the equation

$$
r^{\prime}=a(t) r+b(t) r^{\sigma}
$$

where $a(t)$ and $b(t)$ are continuous and $0<\sigma<1$. Suppose $b(t)>0$ for $t_{0}<t \leqq t_{1}$ and let $L(r)$ be given by $L(r)=1 / r^{\sigma}$. With $w_{1}(t, r)=a_{+}(t) r$, $w_{2}(t, r)=-a_{-}(t) r+b(t) r^{\sigma}$, where $a_{+}(t)=\max \{a(t), 0\}, a_{-}(t)=\max \{-a(t), 0\}$ and $r_{0}>0$ chosen sufficiently small, Theorem 1 implies that the zero solution of (4) is not unique to the right at $t_{0}$ and, hence, not stable at $t_{0}$. We see then that equations of the form of (4) can be used in conjunction with Liapunov functions to obtain standard types of stability only if $b(t) \leqq 0$ (see, for example, [3, Theorem 3.7.8]).

We now give some simple conditions which insure the various standard types of stability.

THEOREM 2. Let (I), (III), and (IV) hold. If for each $t_{0} \geqq 0$ there is a constant $M\left(t_{0}\right)$ so that

$$
\int_{t_{0}}^{t}\left(w_{1}\left(s, r_{0}\right) L\left(r_{0}\right)+q(s)\right) d s \leqq M\left(t_{0}\right)
$$

for all $t \geqq t_{0}$, then $r=0$ is stable. If $M\left(t_{0}\right)$ can be chosen independent of $t_{0}$, then $r=0$ is uniformly stable.

Proof. Let $\varepsilon>0, \varepsilon \leqq r_{0}$, and $t_{0} \geqq 0$ be given. From (1), (I), and (III), we see that if $r(t)$ is a solution of (1),

$$
r^{\prime}(t) L(r(t)) \leqq w_{1}\left(t, r_{0}\right) L\left(r_{0}\right)+q(t)
$$

as long as $r(t) \leqq r_{0}$ and an integration yields

$$
\int_{r\left(t_{0}\right)}^{r(t)} L(r) d r \leqq \int_{t}^{t}\left(w_{1}\left(s, r_{0}\right) L\left(r_{0}\right)+q(s)\right) d s \leqq M\left(t_{0}\right) .
$$

From (IV), it follows that $\delta=\delta\left(t_{0}, \varepsilon\right)>0$ can be chosen so that

$$
\int_{\delta}^{\varepsilon} L(r) d r>M\left(t_{0}\right)
$$


Now if $r\left(t_{0}\right)<\delta$ we see that we must have $r(t)<\varepsilon$ for all $t \geqq t_{0}$. Also, it is obvious that if $M\left(t_{0}\right)=M$, then $\delta=\delta(\varepsilon)$ and the stability is uniform.

THEOREM 3. Let (I), (III), and (IV) hold. If

$$
\int^{\infty}\left(w_{1}\left(s, r_{0}\right) L\left(r_{0}\right)+q(s)\right) d s=-\infty
$$

then the zero solution of (1) is equi-asymptotically stable. If, further, (5) holds with $M\left(t_{0}\right)$ independent of $t_{0}$ and, given $M_{1}<0$, there exists $T=$ $T\left(M_{1}\right)>0$ so that

$$
\int_{t}^{t_{0}+T}\left(w_{1}\left(s, r_{0}\right) L\left(r_{0}\right)+q(s)\right) d s<M_{1}
$$

for every $t_{0} \geqq 0$, then $r=0$ is uniformly asymptotically stable.

Proof. Noting that (6) implies (5) we need only show that if $r(t)$ is a solution of (1) with $r\left(t_{0}\right)<\delta\left(t_{0}, \varepsilon\right)$, then $r(t) \rightarrow 0$ as $t \rightarrow \infty$ and the first statement is proven as asymptotic stability implies equi-asymptotic stability in the scalar case. That $r(t) \rightarrow 0$ follows immediately from (6) as

$$
\int_{r\left(t_{0}\right)}^{r(t)} L(r) d r \leqq \int_{t_{0}}^{t}\left(w_{1}\left(s, r_{0}\right) L\left(r_{0}\right)+q(s)\right) d s \rightarrow-\infty .
$$

To prove the second statement we need only show that $r=0$ is quasiuniformly asymptotically stable as the uniform stability of $r=0$ follows from Theorem 2 .

Suppose $r(t)$ is a solution of (1) with $r\left(t_{0}\right)<\delta\left(r_{0}\right)$ and let $\eta>0$ be given. As $r=0$ is uniformly stable, there exists $\delta_{1}>0$ so that if $r\left(t_{1}\right)<\delta_{1}$ then $r(t)<\eta$ for all $t \geqq t_{1}$. Choose $T=T\left(M_{1}\right)$ where $M_{1}=\int_{r_{0}}^{\delta_{1}} L(r) d r$. Arguing as before, we see that

$$
\int_{r\left(t_{0}\right)}^{r\left(t_{2}\right)} L(r) d r<\int_{r_{0}}^{\delta_{1}} L(r) d r
$$

where $t_{2}=t_{0}+T$ and so $r\left(t_{2}\right)<\delta_{1}$. Hence we have $r(t)<\eta$, for all $t \geqq$ $t_{0}+T$, and the proof is complete.

As an application of Theorems 2 and 3, consider

$$
r^{\prime}=a(t) r^{\alpha}+b(t) r^{\beta}
$$

where $a$ and $b$ are continuous on $[0, \infty)$ and $1 \leqq \alpha<\beta$. Choosing $L(r)=$ $\left(1 / r^{\alpha}\right), w_{1}(t, r)=b_{+}(t) r^{\beta}$, and $w_{2}(t, r)=a(t) r^{\alpha}-b_{-}(t) r^{\beta}$ we see that the zero solution of (7) is stable if

$$
\int_{t_{0}}^{t}\left(a(s)+b_{+}(s) r_{0}^{\beta-\alpha}\right) d s
$$


is bounded for some $r_{0}>0$ and for every $t \geqq t_{0}$. It is equi-asymptotically stable if

$$
\int_{t_{0}}^{\infty}\left(a(s)+b_{+}(s) r_{0}^{\beta-\alpha}\right) d s=-\infty .
$$

ACKNOWLEDGMENT. The author wishes to thank the referee for helpful comments which weakened the hypotheses of Theorems 2 and 3.

\section{REFERENCES}

1. F. Brauer, Some refinements of Lyapunov's second method, Canad. J. Math. 17 (1965), 811-819. MR 31 \#3670.

2. T. G. Hallam and J. W. Heidel, Structure of the solution set of some first order differential equations of comparison type, Trans. Amer. Math. Soc. 160 (1971), 501-512.

3. V. Lakshmikantham and S. Leela, Differential and integral inequalities: Theory and applications. Vol. 1: Ordinary differential equations, Math. in Science and Engineering, vol. 55, Academic Press, New York, 1969.

Department of Mathematics, Southern Illinois University, Carbondale, ILLINOIS 62901 